\title{
Specificity of Small c-Type Cytochromes in Anaerobic Ammonium Oxidation
}

\author{
Mohd. Akram, Josephine Bock, Andreas Dietl,* and Thomas R.M. Barends* \\ Cite This: ACS Omega 2021, 6, 21457-21464 \\ Read Online
}

ABSTRACT: Anaerobic ammonium oxidation (anammox) is a bacterial process in which ammonium and nitrite are combined into dinitrogen gas and water, yielding energy for the cell. This process relies on a series of redox reactions catalyzed by a set of enzymes, with electrons being shuttled to and from these enzymes, likely by small cytochrome $c$ proteins. For this system to work productively, these electron carriers require a degree of specificity toward the various possible redox partners they encounter in the cell. Here, we compare two cytochrome $c$ proteins from the anammox model organism Kuenenia stuttgartiensis. We show that they are highly homologous, are expressed at comparable levels, share the same fold, and display highly similar redox potentials, yet one of them accepts electrons from the metabolic enzyme hydroxylamine oxidase (HAO) efficiently, whereas the other does not. An analysis of the crystal structures supplemented by Monte Carlo simulations of the transient redox interactions suggests that this difference is at least partly due to the electrostatic field surrounding the proteins, illustrating one way in which the electron carriers in anammox could attain the required specificity. Moreover, the simulations suggest a different "outlet" for electrons on HAO than has traditionally been assumed.

\section{INTRODUCTION}

Anaerobic ammonium oxidation (anammox) is a bacterial process in which ammonium $\left(\mathrm{NH}_{4}^{+}\right)$and nitrite $\left(\mathrm{NO}_{2}^{-}\right)$are converted into dinitrogen gas $\left(\mathrm{N}_{2}\right)$ and water, yielding energy for the cell. ${ }^{1}$ The process takes place in a specialized, membrane-enclosed cellular compartment, the anammoxosome, ${ }^{2}$ which contains the redox-active proteins at the heart of the process (Figure 1). The first step is the one-electron reduction of $\mathrm{NO}_{2}^{-}$to nitric oxide (NO) by a nitrite reductase $(\mathrm{NiR}) .^{3-6}$ The resulting $\mathrm{NO}$ is then combined with $\mathrm{NH}_{4}^{+}$to yield the extremely reactive and unusual intermediate hydrazine $\left(\mathrm{N}_{2} \mathrm{H}_{4}\right)$ by the unique hydrazine synthase $(\mathrm{HZS})^{7}$ in a process that takes up a further three electrons. This reaction likely proceeds via hydroxylamine $\left(\mathrm{NH}_{2} \mathrm{OH}\right)$ as an intermediate. It is assumed that any hydroxylamine escaping from HZS is converted back into NO by the octaheme $c$-type cytochrome hydroxylamine oxidase (HAO), ${ }^{8,9}$ releasing three electrons. Finally, the hydrazine is oxidized to $\mathrm{N}_{2}$ by hydrazine dehydrogenase (HDH), ${ }^{9,10}$ releasing four electrons at an extremely low redox potential of $-750 \mathrm{mV}$. Together, these redox reactions fuel an electron transport chain that generates a proton gradient across the anammoxosomal membrane. This proton gradient, in turn, is used to drive ATP synthesis. ${ }^{1}$ Strikingly, NiR, HZS, HAO, and $\mathrm{HDH}$ are all soluble proteins distributed throughout the anammoxosome, ${ }^{11}$ yet the flow of electrons set up by their redox reactions must necessarily pass through the membrane to allow proton translocation. Thus, an efficient electron transport system is required to shuttle electrons between the membrane on the one hand and the various soluble redox proteins on the other hand. Moreover, this system has to be highly specific, as e.g. the low-potential electrons released by $\mathrm{HDH}$ should be transported at least preferentially to the membrane, rather than being shuttled to, for instance, NiR or HZS, since this would result in an effective short-circuit of the system. ${ }^{10}$

As anammox bacteria express a large number of small $c$-type cytochromes, these have been proposed to perform the shuttling of electrons between the various parts of the metabolic machinery. However, few of these have been characterized and little is known about the precise role of the various representatives of this class in the anammox metabolism, including how they recognize their binding partners. Here, we present the structures and biochemical characterization of two of these proteins from the anammox model organism Kuenenia stuttgartiensis: Kustc0562 and highly homologous Kustc0563, which show relatively high transcription levels (Figure S1). Indeed, Kustc0563 was the first protein from an anammox organism to be purified from biomass $^{12}$ and also the first to be heterologously expressed and characterized. ${ }^{13}$ We show that the two proteins are highly similar structurally and electrochemically yet find that Kustc0563 is able to efficiently accept electrons from $K$.

Received: April 30, 2021

Accepted: August 2, 2021

Published: August 9, 2021 


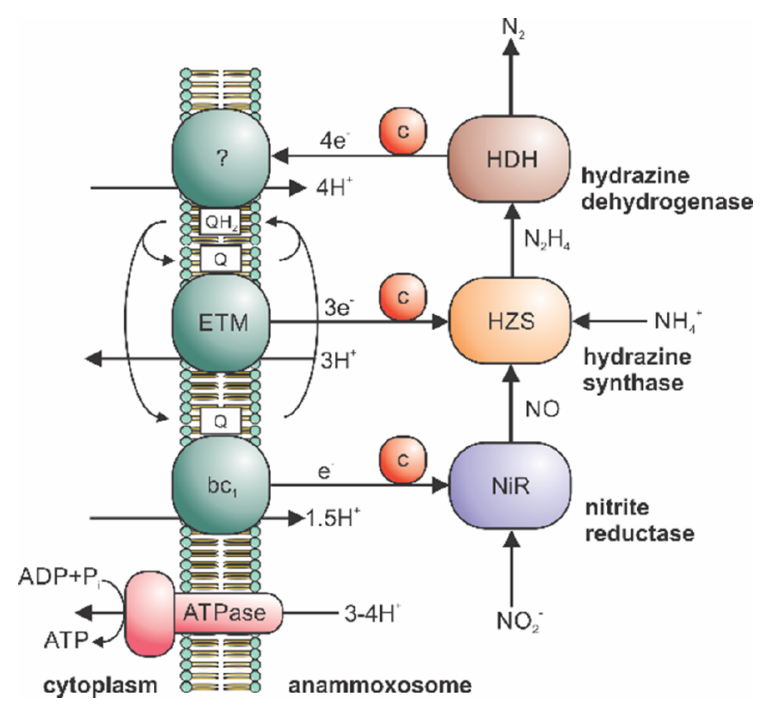

Figure 1. Overview of the central anammox metabolism. An electron transport chain located in the membrane of the anammoxosome is driven by a set of redox reactions catalyzed by NiR, HZS, and HDH. As these are soluble enzymes distributed across the interior of the anammoxosome, a transport system is required to shuttle electrons between these enzymes and the membrane, which is proposed to involve $c$-type cytochromes (red, labeled " $c$ ").

stuttgartiensis HAO (also known as Kustc1061) in hydroxylamine oxidation assays, whereas Kustc0562 does not do so. Moreover, Monte Carlo simulations of the electrostatic interactions indicate that a disparity in their charge distributions contributes to this difference. Moreover, the simulations suggest a different "outlet" for electrons from HAO when interacting with Kustc0563 than that has traditionally been assumed for this class of proteins.

\section{RESULTS}

Kustc0562 and Kustc0563 have Very Similar Structures. Kustc0562 and Kustc0563 share 37\% sequence identity after cleavage of the signal sequence (Figure $2 \mathrm{a}$ ) and display a typical type-I cytochrome $c$ fold (Figure $2 b, c)$. Their crystal structures can be superimposed to an RMSD of $1.2 \AA$ for 81 $\mathrm{C} \alpha$ atoms. In the crystal structure of Kustc0563, the heme iron is proximally coordinated by His47 and distally by Met91; in Kustc0562, the iron is proximally coordinated by the homologous His51, but the distal ligand is an imidazole molecule, presumably from the crystallization solution. Met94, the residue in Kustc0562 homologous to the distal Met in Kustc0563, has moved toward the side of the heme with the propionate groups to make space for imidazole, and a part of the loop containing this residue shows a different conformation than that in Kustc0563. Indeed, when $100 \mathrm{mM}$ imidazole was added to oxidized Kustc0562, the UV-vis spectrum showed a shift of the Soret band from 411 to $406 \mathrm{~nm}$, which was accompanied by the disappearance of a weak charge-transfer band at $690 \mathrm{~nm}$, indicative of methionine coordination to the heme iron ${ }^{14}$ (Figure S2a). When imidazole was removed by buffer exchange, the spectral features of oxidized Kustc0562 were restored. The same effect was observed for Kustc0563 (Figure S2b). In both proteins, the heme surroundings are predominantly hydrophobic.

Kustc0562 and Kustc0563 have Very Similar Redox Potentials. Spectropotentiometry (Figure 2d and S3) revealed that Kustc0562 and Kustc0563 have redox potentials a.

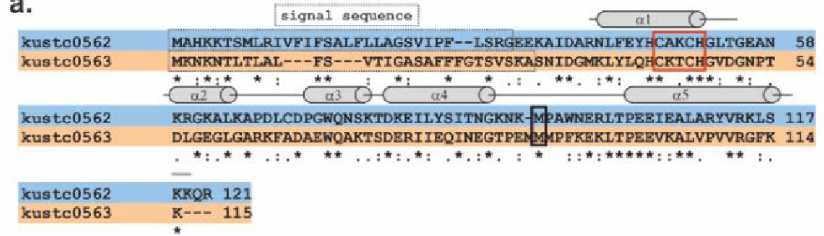

b.

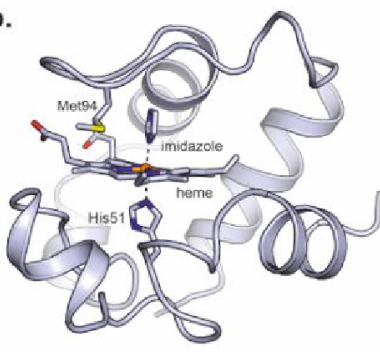

c.
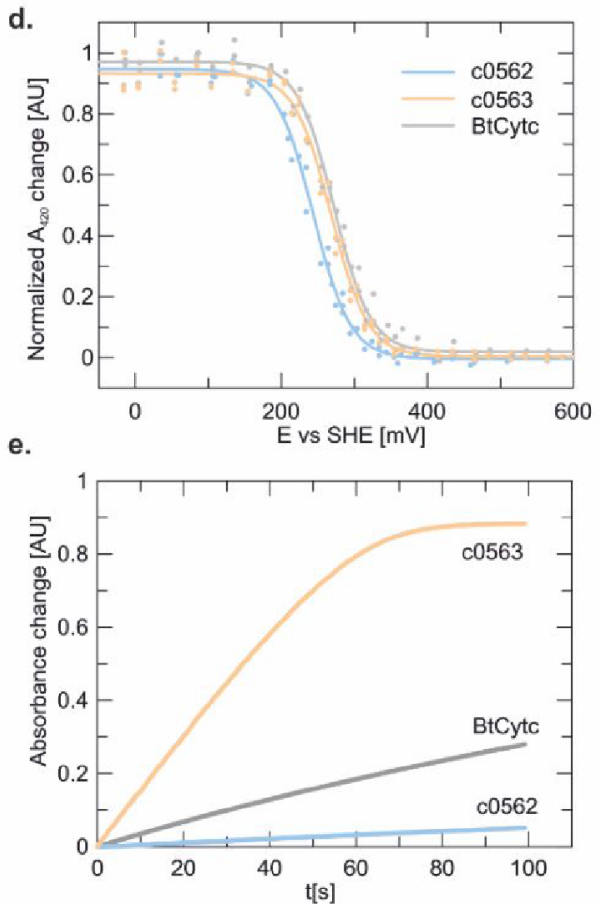

Figure 2. (a) Alignment of the sequences of Kustc0562 and Kustc0563. The signal sequence and the secondary structure elements are shown above the sequence. The heme binding motif and the distal methionine are indicated by the red and black boxes, respectively. (b) Crystal structure of Kustc0562. The protein is shown as a cartoon, with the heme and the iron-coordinating residues shown as sticks. The cysteine residues bound covalently to the heme were omitted for clarity. (c) Crystal structure of Kustc0563 presented as in panel (b). (d) Spectroelectrochemistry of Kustc0562, Kustc0563, and bovine (Bos taurus) cytochrome $c$ (BtCytc). The normalized heme absorbance at $420 \mathrm{~nm}$ is shown as a function of the applied potential vs SHE. (e) Progression curves of hydroxylamine oxidation $(100 \mu \mathrm{M})$ catalyzed by $\mathrm{KsHAO}\left(1.2 \mu \mathrm{g} \mathrm{mL}^{-1}\right)$ using $50 \mu \mathrm{M}$ either Kustc0562, Kustc0563, or BtCytc as the electron acceptor. The absorbance change at $550 \mathrm{~nm}$ is plotted against time starting from substrate addition.

of +244 and $+268 \mathrm{mV}$ versus standard hydrogen electrode (SHE), respectively. These values are similar to the redox potential of bovine mitochondrial cytochrome $c$ (BtCytc), which had earlier been reported to be $+262 \mathrm{mV}$ versus $\mathrm{SHE}^{15}$ and for which we find the value to be $+272 \mathrm{mV}$ versus SHE. Previously, Huston et al. ${ }^{13}$ had found a redox potential of +230 $\mathrm{mV}$ versus SHE for Kustc0563. These values are all very similar 
Table 1. Enzyme Kinetics of Hydroxylamine Oxidation by KsHAO with Various Electron Acceptors ${ }^{a}$

$\begin{array}{ccccc}\text { electron acceptor } & V_{\max } \mathrm{NH}_{2} \mathrm{OH}\left[\mu \mathrm{mol} \mathrm{min}^{-1} \mathrm{mg}^{-1}\right] & K_{\mathrm{m}} \mathrm{NH}_{2} \mathrm{OH}[\mu \mathrm{M}] & \mathrm{k}_{\text {cat }} \mathrm{NH}_{2} \mathrm{OH}\left[\mathrm{s}^{-1}\right]\left(\mathrm{KsHAO}_{\mathrm{H}} \mathrm{trimer}\right) & \mathrm{k}_{\text {cat }} / \mathrm{K}_{\mathrm{m}}\left[\mu \mathrm{M}^{-1} \mathrm{~s}^{-1}\right] \\ \text { Kustc0562 } & \text { n.d. } & \text { n.d. } & \text { n.d. } & \text { n.d. } \\ \text { Kustc0563 } & 15.8 \pm 0.3 & 13.9 \pm 0.7 & 8.4 & 3.5 \\ \text { BtCytc } & 2.7 \pm 0.1 & 5.8 \pm 0.7 & 8.4 & 1.4\end{array}$

${ }^{a}$ With Kustc0562, only marginal activity could be detected and no Michaelis-Menten parameters were determined.

in light of the redox potential of the hydroxylamine/nitric oxide redox pair, which is $-30 \mathrm{mV}$ versus SHE.

Kustc0563 Is Able to Accept Electrons from KsHAO, but the Highly Similar Kustc0562 is Much Less So. We performed assays measuring the rate of hydroxylamine oxidation by KsHAO using Kustc0562, Kustc0563, and BtCytc as electron acceptors (Figure $2 \mathrm{e}$ and $\mathrm{S} 4$ and Table 1). The highest activity was measured using Kustc0563 with a $V_{\max }$ of about 6 times higher than that obtained with BtCytc. Strikingly, however, despite the structural and electrochemical similarities between Kustc0562 and Kustc0563, only marginal activity could be detected when Kustc0562 was used as the electron acceptor.

Monte Carlo Interaction Mapping Suggesting a Binding Interface between Kustc0563 and HAO. To rationalize this difference in ability to accept electrons from $\mathrm{HAO}$, we performed Monte Carlo interaction mapping to identify possible interaction sites between Kustc0563 and HAO using MCMAP 1.0. ${ }^{16}$ This software package allows a ligand protein to randomly move in the electrostatic field of a receptor molecule, resulting in an ensemble of mutual positions and orientations, thus taking the typically transient nature of interactions between redox-active proteins into account. The simulations predict a preferential interaction surface between Kustc0563 and KsHAO (Figure 3a). On Kustc0563, this involves a ring of negatively charged residues on the surface around the heme, in addition to several hydrophobic and two positively charged residues (Figure $3 \mathrm{~b}$ ). On the trimeric KsHAO, which is shaped like a tulip bulb with protrusions on three sides, the predicted interaction surface involves the concave area between two protrusions. This area comprises several positively charged residues, heme 3 of the proteins' multiheme electron transport system (see below), as well as some neutral and hydrophobic residues (Figure 3c,d).

Kustc0562 and Kustc0563 Have Very Different Electrostatics. Inspection of the electrostatics of Kustc0562, Kustc0563, and KsHAO offers an attractive explanation for the observed difference in the ability between Kustc0562 and Kustc0563 to serve as an electron acceptor for HAO in hydroxylamine oxidation reactions. The predicted interaction surface on HAO displays a pronounced positive charge, which would complement the equally pronounced negative charge on the predicted interaction surface on Kustc0563 (Figure 4). In contrast, despite the strong sequence similarity between the two proteins, the corresponding surface on Kustc0562 displays a pronounced positive charge, which would make interactions with the proposed binding site on the HAO surface electrostatically unfavorable.

\section{DISCUSSION}

Although several central anammox metabolic enzymes have been purified and characterized biochemically and/or structurally, ${ }^{3-10}$ little is known about the ways in which these proteins exchange electrons with other parts of the anammox metabolism. It has been suggested that small c-type
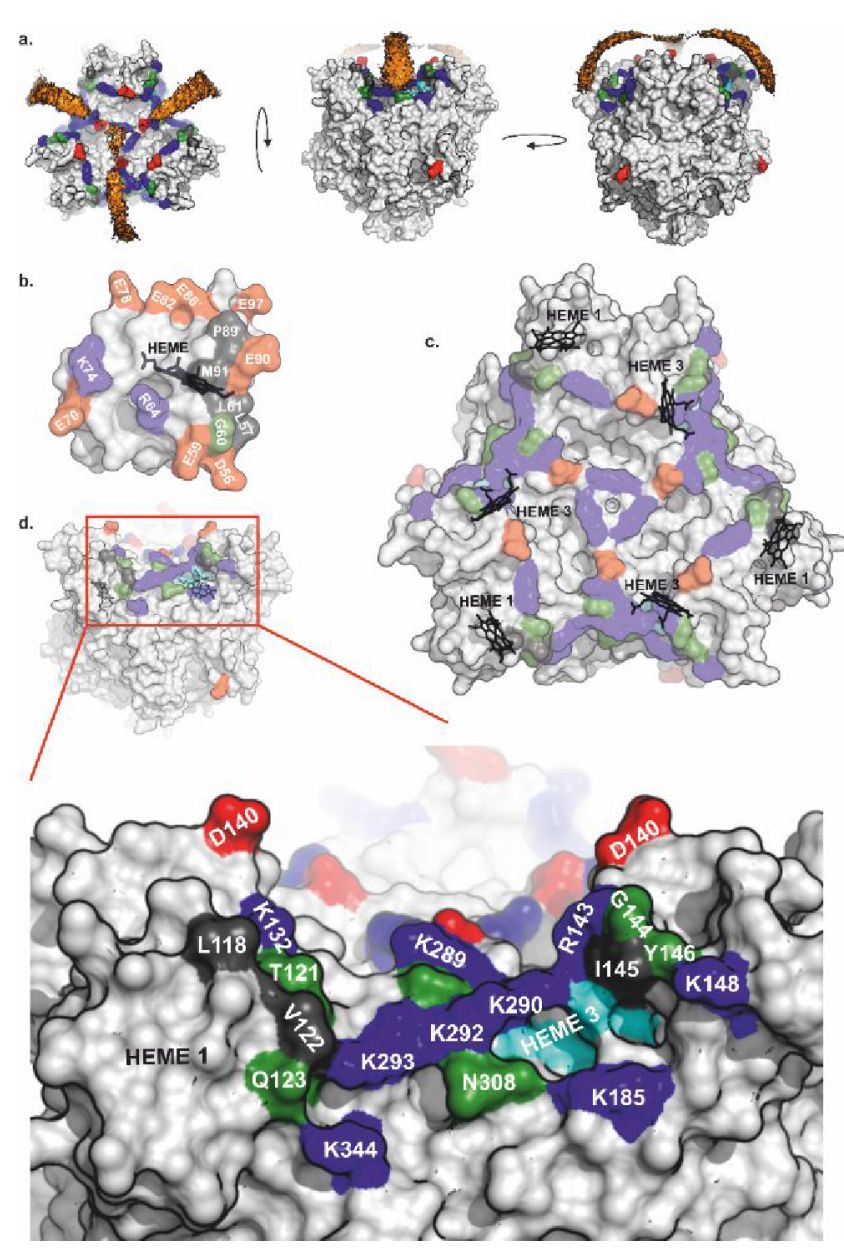

Figure 3. Results of the Monte Carlo mapping of the interaction between KsHAO and Kustc0563. (a) KsHAO is shown in three orientations, with the preferred positions of Kustc0563 during the Monte Carlo run shown in orange. (b) Predicted interaction surface on Kustc0563 (white), with the residues predicted to be involved in the interaction with KsHAO shown in color. The heme is shown as sticks, negatively charged residues are indicated in red, neutral residues are indicated in green, hydrophobic residues are indicated in gray, and positively charged residues are indicated in blue. (c) As (b) for the predicted interaction surface on KsHAO. (d) Closeup of the interaction surface on KsHAO using the same color coding as in panel (b).

cytochromes act as electron shuttles between the soluble redox enzymes of the anammox metabolism on the one hand, and the electron transport chain in the membrane on the other. NaxLS, a stable complex of two small cytochrome $c$ proteins, was found to interact specifically with HZS in pulldown assays, ${ }^{17}$ and a putative cytochrome $c$ binding site was identified on the HZS surface. ${ }^{7}$ Moreover, based on its hollow structure with small entrances to a central cavity, $\mathrm{HDH}$ was suggested to possess a specificity filter, allowing only small proteins to access binding sites for redox partners inside the 
a.
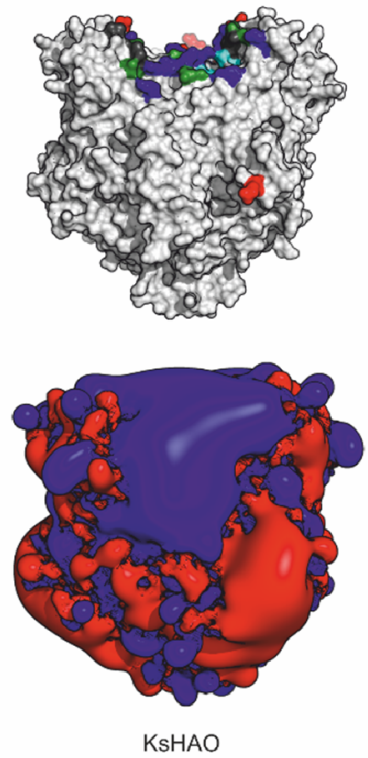

b.

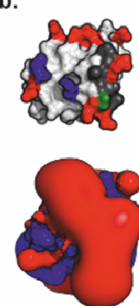

Kustc0563

d.

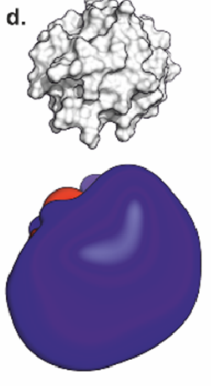

BtCytc c

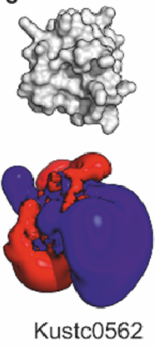

parameters $\left(k_{\text {cat }}=8.4 \mathrm{~s}^{-1}, K_{\mathrm{m}}=5.8 \mu \mathrm{M}\right)$ similar to the values reported previously ${ }^{8}\left(k_{\mathrm{cat}}=15 \mathrm{~s}^{-1}, K_{\mathrm{m}}=4.4 \mu \mathrm{M}\right)$. Importantly, however, the comparison of BtCytc with Kustc0563 shows that the latter protein supports hydroxylamine oxidation by $\mathrm{KsHAO}$ at a rate that is approximately 6 times higher $\left(k_{\mathrm{cat}}=\right.$ $\left.48.4 \mathrm{~s}^{-1}, K_{\mathrm{m}}=13.9 \mu \mathrm{M}\right)$.

Moreover, Kustc0562 supported KsHAO activity only at marginal rates under identical circumstances. The striking contrast in electrostatics around the interaction sites on Kustc0562 and Kustc0563 provides an attractive explanation for this difference. Like Kustc0562, BtCytc also displays a pronounced positive surface electrostatic charge around the heme access site ${ }^{19,20}$ (Figure 4d) and, as expected, given this, BtCytc is indeed a less efficient electron acceptor than Kustc0563 in HAO-catalyzed hydroxylamine oxidation. However, the fact that BtCytc is still able to accept electrons from HAO at a higher rate than Kustc0562 shows that simple electrostatics alone do not fully explain the differing abilities of various proteins to act as an electron acceptor in these reactions, and possibly, BtCytc interacts with KsHAO via a different interaction site than Kustc0563. Nevertheless, the differences in electrostatics between Kustc0562 and Kustc0563 do highlight a mechanism in which the much-needed specificity of electron transport proteins toward their redox partners may at least in part be conferred.

Moreover, it is important to note that the results presented here do not necessarily mean that Kustc0563 is a physiological redox partner for KsHAO. In Nitrosomonas europaea, the highly homologous hydroxylamine oxidoreductase (NeHAO) transfers the electrons derived from hydroxylamine oxidation to a dedicated tetraheme protein, cytochrome c554. ${ }^{21,22}$ The genome of $K$. stuttgartiensis does not, however, encode homologs of this protein, although there are soluble multiheme $c$-type cytochromes that are expressed at high levels such as the tetraheme proteins Kustc1170 and Kuste2854. The latter was recently characterized in detail and found to exchange electrons with HZS. ${ }^{23}$ However, there is no a priori need for the redox partner of KsHAO to be a multiheme protein. As a typical trimeric HAO-like octaheme cytochrome $c$, KsHAO possesses a 24-heme electron relay system to transport electrons between the active site heme 4 of each monomer and electron-transfer sites on the surface of the protein. ${ }^{24}$ This system can store multiple electrons abstracted from a substrate molecule, so that they may be transferred to single heme proteins successively, as confirmed by the activity of KsHAO with single electron acceptors such as Kustc0563 and BtCytc.

On the surface of KsHAO, the predicted site for interactions with Kustc0563 between the protrusions on the tulip-bulb shaped trimer is essentially the same as that proposed for the interaction between the homologous $\mathrm{NeHAO}$ and its cognate redox partner, cytochrome c554, based on docking studies. ${ }^{25}$ Traditionally, these electron-transfer sites are believed to be located at heme 1 of each monomer in the protrusions on the trimer. Binding of a redox partner close to heme 1 was proposed to raise its redox potential, thus "opening a gate" allowing the transfer of an electron to heme 1 and from there to the redox partner. ${ }^{26}$ Moreover, in the related Kustc0457/ Kustc0458 complex, the typical 24-heme electron transport system of HAO-like Kustc0458 is extended from its heme 1 onward with the hemes of the complex partner Kustc0457. ${ }^{3}$ Interestingly, however, in our simulations, heme 3, which in $\mathrm{KsHAO}$ lies at the surface of the protein at the bottom of the concave surface between the protrusions, is consistently 
predicted to be part of the interaction surface with Kustc0563. This opens up the possibility that in the interaction between $\mathrm{KsHAO}$ and Kustc0563, heme 3 is the outlet for electrons rather than heme 1.

\section{MATERIALS AND METHODS}

Protein Preparation. Signal peptides were predicted in both the Kustc0562 and Kustc0563 protein sequences using the Signal 3.0 server, employing the hidden Markov model (HMM). ${ }^{27}$ The corresponding genes were then amplified without their signal peptides by PCR from $K$. stuttgartiensis genomic DNA using primers listed in Table S1. The amplified products were cloned into pUC19kan3 using NotI and XhoI restriction sites. This vector; which adds a tobacco etch virus (TEV) protease-cleavable, N-terminal His-tag to the coding region; uses the periplasmic signal sequence from a tetraheme cytochrome of Shewanella oneidensis $M R-1^{28}$ and is constitutively transcribed under a lac promoter. In addition, Kustc0563 was cloned into pUC19kan2a, which adds a noncleavable C-terminal His-tag. Cells of S. oneidensisMR-1 were then transformed with one of these vectors by electroporation, and the transformants were cultured in LB medium with $50 \mu \mathrm{g} / \mathrm{mL}$ kanamycin using $5 \mathrm{~L}$ nonbaffled flasks shaking at $90-95 \mathrm{rpm}$ and $30{ }^{\circ} \mathrm{C}$. When an OD600 of $0.6-0.8$ was reached, shaking and temperature were reduced to 50-60 $\mathrm{rpm}$ and $20^{\circ} \mathrm{C}$, respectively, and incubation was continued for 60-70 h. The cells were then harvested by centrifugation at $6000 \mathrm{rpm}$ in an F9-6X 1000 LEX rotor (Thermo Scientific, Darmstadt, Germany) for $10 \mathrm{~min}$ at $4{ }^{\circ} \mathrm{C}$ and suspended in a wash/lysis buffer (50 mM TrisCl pH 8.0, $300 \mathrm{mM} \mathrm{NaCl}$, and $10 \mathrm{mM}$ imidazole) at a 1:4 ratio of wet pellet weight to buffer volume. Cell lysis was performed by sonication in $100 \mathrm{~mL}$ portions on ice using a Branson W-450 sonifier (G. Heinemann, Schwäbisch Gmünd, Germany) at 50\% amplitude with $0.5 \mathrm{~s}$ bursts and 10 minutes of total on time. The lysate was then cleared by ultracentrifugation at $160000 \mathrm{~g}$ for $45 \mathrm{~min}$ at $4{ }^{\circ} \mathrm{C}$ in a Ti-45 rotor (Beckman Coulter, Krefeld, Germany). The supernatant was then loaded onto Ni-NTA agarose (Qiagen, Hilden, Germany) or Ni-IDA Profinity beads (BioRad laboratories $\mathrm{GmbH}$, Munich, Germany) pre-equilibrated with wash buffer $(50 \mathrm{mM}$ TrisCl $\mathrm{pH} 8.0,300 \mathrm{mM} \mathrm{NaCl}$, and $10 \mathrm{mM}$ imidazole). The flow-through was reloaded twice, after which the column was washed with 20 volumes of wash buffer. The proteins were then eluted in $50 \mathrm{mM}$ TrisCl $\mathrm{pH} 8.0,300$ $\mathrm{mM} \mathrm{NaCl}$, and $250 \mathrm{mM}$ imidazole. For the constructs with cleavable $\mathrm{N}$-terminal His-tags, protein-containing eluate fractions were buffer-exchanged to TEV digest buffer (20 $\mathrm{mM}$ TrisCl pH 8.0, $150 \mathrm{mM} \mathrm{NaCl}$, and $2 \mathrm{mM}$ TCEP) before being concentrated to $\approx 1 \mathrm{~mL}$ using $3 \mathrm{kDa}$ molecular-weight cutoff (MWCO) Amicon concentrators. TEV digestion was performed overnight at an $A_{280}^{1 \mathrm{~cm}}$ (protein)-to-TEV ratio of 100:1 in a cold room with gentle rotation. The next day, the Ni-NTA chromatography step was repeated to remove any uncleaved protein as well as the His-tagged TEV protease, collecting the flow-through that contained the cleaved protein. This material was again concentrated to $\approx 1 \mathrm{~mL}$ in a $3 \mathrm{kDa}$ MWCO Amicon concentrator. Finally, size-exclusion chromatography (SEC) was performed for all constructs using a Superdex 75 (10/300 GL) column (GE Healthcare, Uppsala, Sweden). The main peak from the SEC was buffer-exchanged using Amicon concentrators to $25 \mathrm{mM} \mathrm{HEPES} / \mathrm{KOH} \mathrm{pH} 7.0$ and $25 \mathrm{mM} \mathrm{KCl}$ and was concentrated to an $A_{280}^{1 \mathrm{~cm}} \sim 6-8$.
Protein purity was assessed by SDS-PAGE followed by heme and Coomassie staining. The identity of the purified proteins was confirmed by peptide mass fingerprinting. Gel slices of the respective protein bands from 15\% SDS-PAGE were excised and digested by trypsin. Stable proteolytic fragments were analyzed by matrix-assisted laser desorption/ionization timeof-flight (MALDI-TOF) mass spectrometry on an Axima TOF2 Performance mass spectrometer (Shimadzu Biotech, Duisburg, Germany) using $\alpha$-cyano-4-hydroxycinnamic acid as the matrix. Characteristic tryptic peptides were identified by MASCOT (Matrix Science Inc., MA, USA). Protein total mass analyses were performed under denaturing conditions by electrospray ionization time-of-flight (ESI-TOF) mass spectrometry on a maXis spectrometer (Bruker Daltonik GmbH, Bremen, Germany). Kustc0562 and Kustc0563 after cleavage of the N-terminal His-tag are designated Kustc0562nt and Kustc0563nt ( $\mathrm{nt}=$ no tag), respectively. C-terminally Histagged Kustc0563 will be designated Kustc0563CT (Cterminal tag). The protein sequences of the expression constructs are given in Figure S5.

UV-Vis Spectroscopy. UV-vis spectra of Kustc0562nt and Kustc0563nt were measured in $200 \mu \mathrm{L}$ quartz microcuvettes (path length $=1 \mathrm{~cm}$, Hellma Analytics, Müllheim, Germany) at $0.5 \mathrm{~nm}$ bandwidth using a Jasco V-650 spectrophotometer (Jasco GmbH, Gross-Umstadt, Germany) equipped with a sample holder at $25{ }^{\circ} \mathrm{C}$. Ferricyanide-oxidized samples of both proteins were diluted to $10 \mu \mathrm{M}$ in a $20 \mathrm{mM}$ potassium phosphate buffer $\mathrm{pH}$ 7.0, and spectra were recorded. A few grains of solid sodium dithionite or $100 \mathrm{mM}$ imidazole were added as appropriate. Raw spectra were baselinecorrected using the Jasco software, and figures were generated using GraFit 7.0.0 (Erithacus Software Ltd, East Grinstead, U.K.).

Enzyme Assays. The protein concentration of $\mathrm{KsHAO}$ was determined by the Bradford method ${ }^{29}$ using a dilution series of a bovine serum albumin standard solution (Thermo Scientific, Darmstadt, Germany) for calibration. HAO assays were performed essentially as described previously ${ }^{8}$ using Kustc0562nt, Kustc0563nt, and bovine cytochrome $c$ (BtCytc, Sigma-Aldrich) as electron acceptors. All electron acceptor cytochromes were fully oxidized by incubation with $10 \mathrm{mM}$ potassium ferricyanide for $30 \mathrm{~min}$ at room temperature followed by buffer exchange to a $20 \mathrm{mM}$ pH 7.0 potassium phosphate buffer using a PD-10 desalting column (GE Healthcare, Uppsala, Sweden). The concentration of the electron acceptor cytochromes was assessed after full reduction with sodium dithionite based on the $\alpha$-band absorbance using an extinction coefficient $\varepsilon_{550}$ of $27.6 \mathrm{mM}^{-1} \mathrm{~cm}^{-1}$ as reported for BtCytc. ${ }^{30}$ For the assays, $0.5 \mathrm{~mL}$ reaction mixtures were prepared, containing $0.6 \mu \mathrm{g}$ of $K$. stuttgartiensis $\mathrm{HAO}$ (KsHAO) and $50 \mu \mathrm{M}$ the appropriate electron acceptor cytochrome in $20 \mathrm{mM}$ potassium phosphate buffer $(\mathrm{pH} 7.0)$ in polystyrene cuvettes. After $1 \mathrm{~min}$ of initial incubation at $37^{\circ} \mathrm{C}$, the reaction was started by adding the appropriate amount of hydroxylamine (1-100 $\mu \mathrm{M}$ final concentration), and the reduction of the electron acceptor was then followed by monitoring the increase in absorbance at $550 \mathrm{~nm}$ using $\Delta \varepsilon_{550}=$ $19.6 \mathrm{mM}^{-1} \mathrm{~cm}^{-1}$ for BtCytc ${ }^{30}$ and $\Delta \varepsilon_{550}=19.1 \mathrm{mM}^{-1} \mathrm{~cm}^{-1}$ for Kustc0563nt. Averaged initial rates from three technical replicate series were fitted by applying the Michaelis-Menten equation. Kinetic parameters are reported in terms of the substrate (hydroxylamine) concentration. 
Redox Potential Determination. An optically transparent thin-layer electrochemical (OTTLE) cell was constructed and operated using a Keithley model 2450 source measure unit (Tektronix Inc., Beaverton, OR, USA) functioning as a potentiostat. To this end, thin $(<1 \mu \mathrm{m})$ gold electrode connections were produced on a $26 \mathrm{~mm} \times 76$ $\mathrm{mm}$ glass microscope slide by spray coating with gold paint (Glanzgold GG B 15/M, Heraeus, Hanau, Germany) and subsequent heating to $520{ }^{\circ} \mathrm{C}$. Moreover, a silver/silver chloride reference electrode patch was prepared on the slide using $\mathrm{Ag} / \mathrm{AgCl}$ ink (ALS Co., Ltd, Tokyo, Japan). A working electrode was prepared using a gold mesh (500 wires/inch, $60 \%$ open area, $10 \mu \mathrm{m}$ thickness; Goodfellow Ltd., Huntingdon, U.K.) that was modified by incubation for $>60$

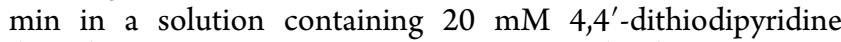
(DTP) in $160 \mathrm{mM} \mathrm{pH} 8.0$ TrisCl and $20 \%$ (v/v) ethanol. For spectroelectrochemical measurements, $18 \mu \mathrm{L}$ of the protein solution in $100 \mathrm{mM} \mathrm{KCl}$ and $10 \mathrm{mM} \mathrm{pH} \mathrm{7.0} \mathrm{MOPS/KOH}$ was mixed with $2 \mu \mathrm{L}$ of a small-molecule mediator mix ( $1 \mathrm{mM}$ each of potassium ferricyanide, $p$-benzoquinone, 2,5-dimethyl- $p$ benzoquinone, 1,2-naphtoquinone, phenazine methosulfate, 1,4-napthoquinone, phenazine ethosulfate, 5-hydroxy-1,4napthoquinone, 2-methyl-1,4-napthoquinone, 2,5-dihydroxyp-benzoquinone, 2-hydroxy-1,4-napthoquinone, anthraquinone, sodium anthraquinone-2-sulphonate, benzyl viologen, and methyl viologen). The protein solution was then pipetted onto the working electrode that was placed on the gold electrodes on the slide and covered with a standard $22 \mathrm{~mm} \times$ $22 \mathrm{~mm}$ glass microscope cover slip. After sealing with parafilm, the cell was mounted in the beam of a Jasco V-760 spectrophotometer (Jasco GmbH, Gross-Umstadt, Germany). The Keithley source meter was used to set potentials and measure currents and also controlled data acquisition by the spectrophotometer using a custom-built interface. Samples were prepoised at $-300 \mathrm{mV}$ for $10 \mathrm{~min}$ and then oxidized to $+300 \mathrm{mV}$, after which they were reduced back again to -300 $\mathrm{mV}$ in $20-60 \mathrm{mV}$ steps. UV-vis spectra were recorded using wavelengths ranging from 350 to $700 \mathrm{~nm}(90 \mathrm{~s} /$ spectrum at $400 \mathrm{~nm} / \mathrm{min}$ ). Raw spectra were baseline-corrected by setting the absorption at $700 \mathrm{~nm}$ to zero. Absorbance values at 420 $\mathrm{nm}$ (the Soret band) were fitted to a Nernstian function using nonlinear least-squares minimization in Microsoft Excel to determine the midpoint potential $E_{\mathrm{m}}$ :

$$
Y=A-A_{\mathrm{ox}}=\frac{a}{\mathrm{e}^{\left((n F / R T)\left(E-E_{\mathrm{m}}\right)\right)}+1}+\text { offset }
$$

where $A$ is the absorbance, $A_{\mathrm{ox}}$ is the absorbance of the fully oxidized state, $a$ is the amplitude, $E$ is the applied potential, $E_{\mathrm{m}}$ is the midpoint potential, and $n$ is the number of electrons $(n=$ 1). For each protein, data from two individual experiments were fitted together. To remove hysteresis effects likely resulting from protein denaturation, the oxidative and reductive limbs of each data series were normalized individually. Spectra for one of each of the data series for Kustc0562 and Kustc0563 are given in Supporting Information Figure S3. The Faraday constant $F$ was taken to be $96485.34 \mathrm{~J}$ $\mathrm{V}^{-1} \mathrm{~mol}^{-1}$, the temperature $T$ was $293 \mathrm{~K}$, and the ideal gas constant $R$ was taken to be $8.3145 \mathrm{~J} \mathrm{~mol}^{-1} \mathrm{~K}^{-1}$. To correct the potential against the SHE, the voltage between the $\mathrm{Ag} / \mathrm{AgCl}$ patch in a drop of $10 \mathrm{mM} \mathrm{MOPS} / \mathrm{KOH}(\mathrm{pH} \mathrm{7.0)}$ and 100 $\mathrm{mM} \mathrm{KCl}$ and a commercial $\mathrm{Ag} / \mathrm{AgCl} / 4 \mathrm{M} \mathrm{KCl}$ reference electrode (Pine Research Instrumentation, Durham, NC, USA; $E^{\circ}=+200 \mathrm{mV}$ vs SHE) was measured.
Crystallization, Data Collection, and Structure Solution. Kustc0562nt was crystallized in $1+1 \mu \mathrm{L}$ hanging drop setups equilibrating against $0.2 \mathrm{M} \mathrm{NaCl}, 30 \%$ (w/v) PEG 8000, $0.1 \mathrm{M}$ imidazole $\mathrm{pH} 8.0$, and $0.277 \mathrm{mM}$ cyclohexylethanoyl- $N$-hydroxyethylglucamide (C-HEGA-8). Flattened needles of up to $400 \mu \mathrm{m}$ in length grew within 2 days at room temperature. These were cryoprotected by soaking in reservoir solution supplemented with $25 \%$ (v/v) ethylene glycol prior to flash-cooling in liquid nitrogen. A $3.1 \AA$ resolution data set was collected on a Rigaku MicroMax 007HF rotating anode equipped with a MAR345 image plate. Kustc0563CT was crystallized in $1+1 \mu \mathrm{L}$ hanging drop setups equilibrating against $15 \%(\mathrm{w} / \mathrm{v})$ PEG $6000,0.5 \mathrm{M} \mathrm{LiCl}$, and $0.1 \mathrm{M}$ sodium cacodylate ( $\mathrm{pH} 6.5$ ), with $0.2 \mu \mathrm{L}$ of $80 \mathrm{mM}$ CHAPS added to the drop. These were cryoprotected by soaking in mother liquor supplemented with $20-25 \%$ (v/v) ethylene glycol and flash-cooled in liquid nitrogen. A high redundancy SAD dataset was collected at $100 \mathrm{~K}$ and $1.7433 \AA$ wavelength at beamline X10SA at the Swiss Light Source (SLS) of the Paul Scherrer Institute (Villigen, Switzerland). Bipyramidally shaped crystals of KustC0563nt were obtained from $0.2 \mathrm{M}$ zinc acetate and 20\% (w/v) PEG 3350 in 96-well sitting drop vapor diffusion setups with a drop size of $100 \mathrm{nl}+$ $100 \mathrm{nl}$, with the concentration of protein being such that $A_{280}^{1 \mathrm{~cm}}=6-7$. Crystals of about $150 \times 100 \mu \mathrm{m}^{2}$ grew in $4-6$ days and were flash-frozen in liquid nitrogen using $20 \%(\mathrm{v} / \mathrm{v})$ ethylene glycol in the reservoir solution as a cryoprotectant. High-resolution diffraction data were collected from these crystals at beamline X10SA at the SLS of the Paul Scherrer Institute (Villigen, Switzerland) at $100 \mathrm{~K}$. All diffraction data were processed with $\mathrm{XDS},{ }^{31}$ and data statistics are reported in Table S2.

Structure Determination and Refinement. The Kustc0563CT diffraction data were phased by single-wavelength anomalous diffraction $(\mathrm{SAD})$ using phenix.autosol, ${ }^{32,33}$ which identified six iron sites indicating six molecules in the asymmetric unit. An initial structure was built automatically, which was completed and refined by iterative cycles of rebuilding in $\mathrm{COOT}^{34}$ and refinement by REFMAC5. ${ }^{35}$ The Kustc0563nt data were phased by molecular replacement using MOLREP $^{36}$ using a monomer from the KustC0563CT structure as the search model. The final model was refined using REFMAC5. ${ }^{35}$ The Kustc0562nt data were phased by molecular replacement using Phaser, ${ }^{37,38}$ using the Kustc0563nt structure as the search model, and refined using REFMAC $^{35}$ and phenix.refine. ${ }^{32}$

Monte Carlo Interaction Site Mapping. Structures were prepared by adding hydrogen and assigning charges with the PDB2PQR server ${ }^{39-41}$ using the PARSE force field ${ }^{42}$ and PROPKA $^{43}$ to assign protonation states at $\mathrm{pH}$ 7.0. For $c$-type heme moieties, parameters were adapted from the PARSE force field as in the work of Akram et al. ${ }^{10}$ Electrostatic potentials were calculated using APBS $3.0^{44}$ assuming a $20 \mathrm{mM}$ potassium phosphate buffer, and Monte Carlo mapping was done with MCMAP 1.0, ${ }^{16}$ performing 1000000 runs of 25000 steps each at a temperature of $300 \mathrm{~K}$ and using a maximum center-of-mass distance of $500 \AA$. Results were collated and visualized using the PyCoALA toolbox. The calculations were performed with various combinations of heme states: all ferric, all ferrous, all ferrous on KsHAO but the heme in Kustc0563 ferric, or all hemes in the ferric state but with an electron on either heme 3 or heme 1 of KsHAO. All 
calculations resulted in essentially the same interaction surface. However, because the calculation with all hemes in the ferrous state identified the most residues in the interaction surfaces, the results from this calculation are shown in Figure 3.

\section{ASSOCIATED CONTENT}

\section{SI Supporting Information}

The Supporting Information is available free of charge at https://pubs.acs.org/doi/10.1021/acsomega.1c02275.

Figures including transcription patterns of Kustc0562/ 63, UV-vis spectra and raw spectroelectrochemistry data of Kustc0562nt and Kustc053nt, MichaelisMenten kinetic data, and protein sequences of the expression constructs used in this study; tables including protein crystal structures and crystallographic data deposited in the protein data bank under entry codes $5 \mathrm{mxy}$ and $7 \mathrm{o} 38$ (PDF)

\section{AUTHOR INFORMATION}

\section{Corresponding Authors}

Andreas Dietl - Department of Biomolecular Mechanisms, Max Planck Institute for Medical Research, D-69120 Heidelberg, Germany; Email: andreas.dietl@mr.mpg.de

Thomas R.M. Barends - Department of Biomolecular Mechanisms, Max Planck Institute for Medical Research, D69120 Heidelberg, Germany; 이이이.org/0000-0002-94883005; Email: thomas.barends@mr.mpg.de

\section{Authors}

Mohd. Akram - Department of Biomolecular Mechanisms, Max Planck Institute for Medical Research, D-69120 Heidelberg, Germany

Josephine Bock - Department of Biomolecular Mechanisms, Max Planck Institute for Medical Research, D-69120 Heidelberg, Germany

Complete contact information is available at:

https://pubs.acs.org/10.1021/acsomega.1c02275

\section{Author Contributions}

M.A. and J.B. expressed and purified proteins, determined crystal structures, and performed biochemical assays. A.D. performed electrochemical and biochemical assays. The manuscript was written through contributions of all authors.

\section{Funding}

This work was funded by the Max Planck Society.

\section{Notes}

The authors declare no competing financial interest.

\section{ACKNOWLEDGMENTS}

The authors thank Boran Kartal, Christina Ferousi, and Joachim Reimann, Radboud University Nijmegen, for the generous gift of KsHAO. They are grateful to Chris Roome for excellent computing support at all times and Melanie Müller for MALDI-TOF analysis. They thank Kerstin-Anikó Seifert and Daniela Odenthal for helping with biochemical assays during the revision process. They are also grateful to Jochen Reinstein for helping in calculating the kinetic parameters. Furthermore, they thank Ilme Schlichting for continuous support and the beamline staff at the Swiss Light Source for their help and excellent facilities.

\section{ABBREVIATIONS}

Tris

TCEP

$\mathrm{k}_{\mathrm{B}}$

PEG

MOPS

NTA

IDA

and SDS-PAGE 2-amino-2-hydroxymethyl-propane-1,3-diol tris(2-carboxyethyl)phosphine

Boltzmann's constant 3-morpholinopropane-1-sulfonic acid nitrilotriacetic acid iminodiacetic acid sodium dodecyl sulfate polyacrylamide gel electrophoresis polyethylene glycol

\section{REFERENCES}

(1) Kartal, B.; De Almeida, N. M.; Maalcke, W. J.; Op Den Camp, H. J. M.; Jetten, M. S. M.; Keltjens, J. T. How to make a living from anaerobic ammonium oxidation. FEMS Microbiol. Rev. 2013, 37, 428461.

(2) Van Niftrik, L. A.; Fuerst, J. A.; Sinninghe Damsté, J. S.; Kuenen, J. G.; Jetten, M. S. M.; Strous, M. The anammoxosome: An intracytoplasmic compartment in anammox bacteria. FEMS Microbiol. Lett. 2004, 233, 7-13.

(3) Dietl, A.; Maalcke, W. J.; Ferousi, C.; Jetten, M. S. M.; Kartal, B.; Barends, T. R. M. A 60-heme reductase complex from an anammox bacterium shows an extended electron transfer pathway. Acta Crystallogr., Sect. D: Biol. Crystallogr. 2019, 75, 333-341.

(4) Ferousi, C.; Schmitz, R. A.; Maalcke, W. J.; Lindhoud, S.; Versantvoort, W.; Jetten, M. S. M.; Reimann, J.; Kartal, B. Characterization of a nitrite-reducing octaheme hydroxylamine oxidoreductase that lacks the tyrosine crosslink. J. Biol. Chem. 2021, 296, No. 100476.

(5) Hira, D.; Matsumura, M.; Kitamura, R.; Furukawa, K.; Fujii, T. Unique hexameric structure of copper-containing nitrite reductase of an anammox bacterium KSU-1. Biochem. Biophys. Res. Commun. 2020, $526,654-660$.

(6) Hira, D.; Toh, H.; Migita, C. T.; Okubo, H.; Nishiyama, T.; Hattori, M.; Furukawa, K.; Fujii, T. Anammox organism KSU-1 expresses a NirK-type copper-containing nitrite reductase instead of a NirS-type with cytochrome cd(1). FEBS Lett. 2012, 586, 1658-1663.

(7) Dietl, A.; Ferousi, C.; Maalcke, W. J.; Menzel, A.; De Vries, S.; Keltjens, J. T.; Jetten, M. S. M.; Kartal, B.; Barends, T. R. M. The inner workings of the hydrazine synthase multiprotein complex. Nature 2015, 527, 394-397.

(8) Maalcke, W. J.; Dietl, A.; Marritt, S. J.; Butt, J. N.; Jetten, M. S. Keltjens, J. T.; Barends, T. R.; Kartal, B. Structural basis of biological NO generation by octaheme oxidoreductases. J. Biol. Chem. 2014, $289,1228-1242$.

(9) Maalcke, W. J.; Reimann, J.; De Vries, S.; Butt, J. N.; Dietl, A.; Kip, N.; Mersdorf, U.; Barends, T. R. M.; Jetten, M. S. M.; Keltjens, J. T.; Kartal, B. Characterization of anammox hydrazine dehydrogenase, a key $\mathrm{N}_{2}$-producing enzyme in the global nitrogen cycle. J. Biol. Chem. 2016, 291, 17077-17092.

(10) Akram, M.; Dietl, A.; Mersdorf, U.; Prinz, S.; Maalcke, W.; Keltjens, J.; Ferousi, C.; De Almeida, N. M.; Reimann, J.; Kartal, B.; Jetten, M. S. M.; Parey, K.; Barends, T. R. M. A 192-heme electron transfer network in the hydrazine dehydrogenase complex. Sci. Adv. 2019, 5, No. eaav4310.

(11) De Almeida, N. M.; Neumann, S.; Mesman, R. J.; Ferousi, C.; Keltjens, J. T.; Jetten, M. S. M.; Kartal, B.; Van Niftrik, L. Immunogold localization of key metabolic enzymes in the anammoxosome and on the tubule-like structures of Kuenenia stuttgartiensis. J. Bacteriol. 2015, 197, 2432-2441.

(12) Cirpus, I. E. Y.; Been, M. D.; Den Camp, H. J. M. O.; Strous, M.; Le Paslier, D.; Kuenen, G. J.; Jetten, M. S. M. A new soluble 10 $\mathrm{kDa}$ monoheme cytochrome c-552 from the anammox bacterium candidatus "Kuenenia stuttgartiensis". FEMS Microbiol. Lett. 2005, 252, $273-278$

(13) Huston, W. M.; Harhangi, H. R.; Leech, A. P.; Butler, C. S.; Jetten, M. S. M.; Op Den Camp, H. J. M.; Moir, J. W. B. Expression 
and characterisation of a major c-type cytochrome encoded by gene kustc0563 from Kuenenia stuttgartiensis as a recombinant protein in Escherichia coli. Protein Expression Purif. 2007, 51, 28-33.

(14) Shechter, E.; Saludjian, P. Conformation of ferricytochrome $c$. IV. Relationship between optical absorption and protein conformation. Biopolymers 1967, 5, 788-790.

(15) Raphael, A. L.; Gray, H. B. Semisynthesis of axial-ligand (position-80) mutants of cytochrome-c. J. Am. Chem. Soc. 1991, 113, $1038-1040$.

(16) Foerster, J. M.; Poehner, I.; Ullmann, G. M. MCMap-A computational tool for mapping energy landscapes of transient protein-protein interactions. ACS Omega 2018, 3, 6465-6475.

(17) Akram, M.; Reimann, J.; Dietl, A.; Menzel, A.; Versantvoort, W.; Kartal, B.; Jetten, M. S. M.; Barends, T. R. M. A nitric oxidebinding heterodimeric cytochrome $c$ complex from the anammox bacterium Kuenenia stuttgartiensis binds to hydrazine synthase. J. Biol. Chem. 2019, 294, 16712-16728.

(18) Hira, D.; Kitamura, R.; Nakamura, T.; Yamagata, Y.; Furukawa, K.; Fujii, T. Anammox organism KSU-1 expresses a novel his/DOPA ligated cytochrome c. J. Mol. Biol. 2018, 430, 1189-1200.

(19) Koppenol, W. H.; Vroonland, C. A. J.; Braams, R. Electricpotential field around cytochrome-c and effect of ionic-strength on reaction-rates of horse cytochrome-c. Biochim. Biophys. Acta 1978, 503, 499-508.

(20) Mirkin, N.; Jaconcic, J.; Stojanoff, V.; Moreno, A. High resolution X-ray crystallographic structure of bovine heart cytochrome $\mathrm{c}$ and its application to the design of an electron transfer biosensor. Proteins 2008, 70, 83-92.

(21) Yamanaka, T.; Shinra, M. Cytochrome c-552 and cytochrome c-554 derived from Nitrosomonas europaes-purification, properties, and their function in hydroxylamine oxidation. J. Biochem. 1974, 75, $1265-1273$.

(22) Andersson, K. K.; Lipscomb, J. D.; Valentine, M.; Munck, E.; Hooper, A. B. Tetraheme cytochrome c-554 from Nitrosomonas europaea-heme-heme interactions and ligand-binding. J. Biol. Chem. 1986, 261, 1126-1138.

(23) Ferousi, C.; Lindhoud, S.; Baymann, F.; Hester, E. R.; Reimann, J.; Kartal, B. Discovery of a functional, contracted heme-binding motif within a multiheme cytochrome. J. Biol. Chem. 2019, 294, 1695316965.

(24) Igarashi, N.; Moriyama, H.; Fujiwara, T.; Fukumori, Y.; Tanaka, $\mathrm{N}$. The $2.8 \AA$ structure of hydroxylamine oxidoreductase from a nitrifying chemoautotrophic bacterium, Nitrosomonas europaea. Nat. Struct. Biol. 1997, 4, 276-284.

(25) Cedervall, P.; Hooper, A. B.; Wilmot, C. M. Structural studies of hydroxylamine oxidoreductase reveal a unique heme cofactor and a previously unidentified interaction partner. Biochemistry 2013, 52, $6211-6218$

(26) Kurnikov, I. V.; Ratner, M. A.; Pacheco, A. A. Redox equilibria in hydroxylamine oxidoreductase. Electrostatic control of electron redistribution in multielectron oxidative processes. Biochemistry 2005, 44, 1856-1863.

(27) Bendtsen, J. D.; Nielsen, H.; Von Heijne, G.; Brunak, S. Improved prediction of signal peptides: SignalP 3.0. J. Mol. Biol. 2004, 340, 783-795.

(28) Heun, M.; Binnenkade, L.; Kreienbaum, M.; Thormann, K. M. Functional specificity of extracellular nucleases of Shewanella oneidensis MR-1. Appl. Environ. Microbiol. 2012, 78, 4400-4411.

(29) Bradford, M. M. A rapid and sensitive method for the quantitation of microgram quantities of protein utilizing the principle of protein-dye binding. Anal. Biochem. 1976, 72, 248-254.

(30) Yonetani, T. Studies on cytochrome $c$ peroxidase.2. Stoichiometry between enzyme $\mathrm{H}_{2} \mathrm{O}_{2}$ and ferrocytochrome $c$ and enzymic determination of extinction coefficients of cytochrome $c$. J. Biol. Chem. 1965, 240, 4509-4514.

(31) Kabsch, W. XDS. Acta Crystallogr., Sect. D: Biol. Crystallogr. 2010, 66, 125-132.

(32) Adams, P. D.; Afonine, P. V.; Bunkoczi, G.; Chen, V. B.; Davis, I. W.; Echols, N.; Headd, J. J.; Hung, L. W.; Kapral, G. J.; Grosse-
Kunstleve, R. W.; McCoy, A. J.; Moriarty, N. W.; Oeffner, R.; Read, R. J.; Richardson, D. C.; Richardson, J. S.; Terwilliger, T. C.; Zwart, P. H. PHENIX: A comprehensive python-based system for macromolecular structure solution. Acta Crystallogr., Sect. D: Biol. Crystallogr. 2010, 66, 213-221.

(33) Adams, P. D.; Afonine, P. V.; Bunkoczi, G.; Chen, V. B.; Echols, N.; Headd, J. J.; Hung, L. W.; Jain, S.; Kapral, G. J.; Kunstleve, R. W. G.; McCoy, A. J.; Moriarty, N. W.; Oeffner, R. D.; Read, R. J.; Richardson, D. C.; Richardson, J. S.; Terwilliger, T. C.; Zwart, P. H. The phenix software for automated determination of macromolecular structures. Methods 2011, 55, 94-106.

(34) Emsley, P.; Cowtan, K. Coot: Model-building tools for molecular graphics. Acta Crystallogr., Sect. D: Biol. Crystallogr. 2004, $60,2126-2132$.

(35) Murshudov, G. N.; Skubak, P.; Lebedev, A. A.; Pannu, N. S.; Steiner, R. A.; Nicholls, R. A.; Winn, M. D.; Long, F.; Vagin, A. A. REFMAC5 for the refinement of macromolecular crystal structures. Acta Crystallogr., Sect. D: Biol. Crystallogr. 2011, 67, 355-367.

(36) Vagin, A.; Teplyakov, A. MOLREP: An automated program for molecular replacement. J. Appl. Crystallogr. 1997, 30, 1022-1025.

(37) McCoy, A. J. Solving structures of protein complexes by molecular replacement with phaser. Acta Crystallogr., Sect. D: Biol. Crystallogr. 2007, 63, 32-41.

(38) McCoy, A. J.; Grosse-Kunstleve, R. W.; Adams, P. D.; Winn, M. D.; Storoni, L. C.; Read, R. J. Phaser crystallographic software. J. Appl. Crystallogr. 2007, 40, 658-674.

(39) Dolinsky, T. J.; Czodrowski, P.; Li, H.; Nielsen, J. E.; Jensen, J. H.; Klebe, G.; Baker, N. A. PDB2PQR: Expanding and upgrading automated preparation of biomolecular structures for molecular simulations. Nucleic Acids Res. 2007, 35, W522-W525.

(40) Dolinsky, T. J.; Nielsen, J. E.; McCammon, J. A.; Baker, N. A. PDB2PQR: An automated pipeline for the setup of PoissonBoltzmann electrostatics calculations. Nucleic Acids Res. 2004, 32, W665-W667.

(41) Unni, S.; Huang, Y.; Hanson, R. M.; Tobias, M.; Krishnan, S.; Li, W. W.; Nielsen, J. E.; Baker, N. A. Web servers and services for electrostatics calculations with APBS and PDB2PQR. J. Comput. Chem. 2011, 32, 1488-1491.

(42) Sitkoff, D.; Sharp, K. A.; Honig, B. Accurate calculation of hydration free-energies using macroscopic solvent models. J. Phys. Chem. 1994, 98, 1978-1988.

(43) Rostkowski, M.; Olsson, M. H. M.; Sondergaard, C. R.; Jensen, J. H. Graphical analysis of $\mathrm{pH}$-dependent properties of proteins predicted using PROPKA. BMC Struct. Biol. 2011, 11, No. 6.

(44) Jurrus, E.; Engel, D.; Star, K.; Monson, K.; Brandi, J.; Felberg, L. E.; Brookes, D. H.; Wilson, L.; Chen, J. H.; Liles, K.; Chun, M. J.; Li, P.; Gohara, D. W.; Dolinsky, T.; Konecny, R.; Koes, D. R.; Nielsen, J. E.; Head-Gordon, T.; Geng, W. H.; Krasny, R.; Wei, G. W.; Holst, M. J.; McCammon, J. A.; Baker, N. A. Improvements to the APBS biomolecular solvation software suite. Protein Sci. 2018, 27, $112-128$. 